\title{
Improved Slope Estimation for SAR Doppler Ambiguity Resolution
}

\author{
Ian G. Cumming, Senior Life Member, IEEE, and Shu Li
}

\begin{abstract}
The idea of using the Radon transform to measure the alignment of linear features in synthetic aperture radar (SAR) data has breathed new life into the "look displacement" class of Doppler ambiguity resolution algorithms. In these algorithms, the slope of target energy is estimated to obtain the satellite beam pointing angle accurately enough to resolve the Doppler ambiguity. After explaining the method and adding some minor improvements, it is shown how it can work well on satellite SAR data. Then, an alternate method is developed that combines the ideas of the Radon and look displacement algorithms to obtain a computationally simpler and more accurate algorithm. In addition, the quality checks of the "spatial diversity" approach are used to increase the robustness of the algorithm. Even though the algorithm was conceived for high-contrast scenes, it works remarkably well in low to medium contrast scenes as well.
\end{abstract}

Index Terms-Doppler ambiguity resolution, Doppler centroid estimation, Radon transform, synthetic aperture radar (SAR) antenna pointing angle.

\section{INTRODUCTION}

I $\mathrm{N}$ high-quality synthetic aperture radar (SAR) processing, the estimation of the Doppler centroid frequency is an essential procedure for good image focus. Due to the fact that the azimuth data are sampled by the pulse repetition frequency (PRF), the Doppler centroid estimate must be expressed in two parts: the baseband Doppler centroid and the Doppler ambiguity, and separate estimators are needed for each part. In the baseband Doppler centroid estimation, several algorithms, such as the "spectral fit" and average cross correlation (ACCC) methods, can give reliable estimates in most cases-usually better than $1 \%$ of the PRF [1]. In solving for the Doppler ambiguity number, a number of algorithms have been used, such as look displacement [2], multiple PRF [3], wavelength diversity (WDA) [4], multilook cross correlation (MLCC), and multilook beat frequency (MLBF) algorithms [5]. However, the accuracy and robustness of the Doppler ambiguity number estimate still needs improvement to satisfy current high-quality SAR processing requirements.

The "look displacement" algorithm proposed in 1986 [2] uses the fact that the average slope of the target trajectory before range cell migration correction (RCMC) is proportional to the beam squint angle and the Doppler centroid. In this method, the Doppler ambiguity is estimated by measuring the range displacement of targets between two azimuth looks

Manuscript received May 20, 2005; revised October 25, 2005.

The authors are with the Department of Electrical and Computer Engineering, The University of British Columbia, Vancouver, BC V6T 1Z4, Canada (e-mail: ianc@ece.ubc.ca; shul@ece.ubc.ca).

Digital Object Identifier 10.1109/TGRS.2005.861925 or, equivalently, by measuring the slope of targets in the range-compressed image.

The Radon transform is a well-known method of detecting linear features in an image [6], such as the slope of lines (Sections I-A and II). Kong et al. have applied the Radon transform to estimate the Doppler centroid frequency of airborne SAR data in 2005 [7]. In the present paper, we adapt Kong's method to satellite SAR data (Section III-A) and propose some algorithm improvements (Section III-B).

Then, a simpler method is introduced that uses an integration rather than the more complicated Radon transform (Section IV). We use a diverse RADARSAT-1 scene to illustrate the effectiveness of the two algorithms in resolving the Doppler ambiguity (Section V). We find that both methods are better than previous established methods and that the new method is slightly better than the Radon method. We conclude that both the Radon method and the new method not only obtain reliable Doppler ambiguity estimates in scenes with bright isolated targets, but also work well in the areas with low to medium contrast (Section VI).

\section{A. Geometry of a SAR Target-Range Cell Migration Slope}

The Doppler centroid is an important parameter in SAR processing. It corresponds to the azimuth or Doppler frequency when the target is illuminated by the center of the beam. A Doppler centroid error can lead to defocusing, lower signal-tonoise ratio (SNR), misregistration, and ambiguities in the processed image.

The Doppler centroid can be derived from a geometry model of the SAR system, with an accurate knowledge of system parameters such as the satellite attitude (see [1, App. 12A]). The geometry model of a SAR system is shown in Fig. 1, where the effective SAR forward velocity is $V_{r}$, and $\theta_{r, c}$ is the beam squint angle measured in the slant range plane. If we know the parameter values, the total Doppler centroid can be obtained from

$$
f_{\eta_{c}}=\frac{2 V_{r}}{\lambda_{c}} \sin \theta_{r, c}
$$

where $\lambda_{c}$ is the wavelength corresponding to the radar carrier frequency. However, as the attitude measurements are usually not accurate enough for precision processing, estimators based on the received data are widely used to obtain Doppler centroid estimates.

A nonzero squint angle, $\theta_{r, c}$, leads to a nonzero average range migration in the range/azimuth plane. If the slope of this average migration can be estimated, the squint angle and thereby the 


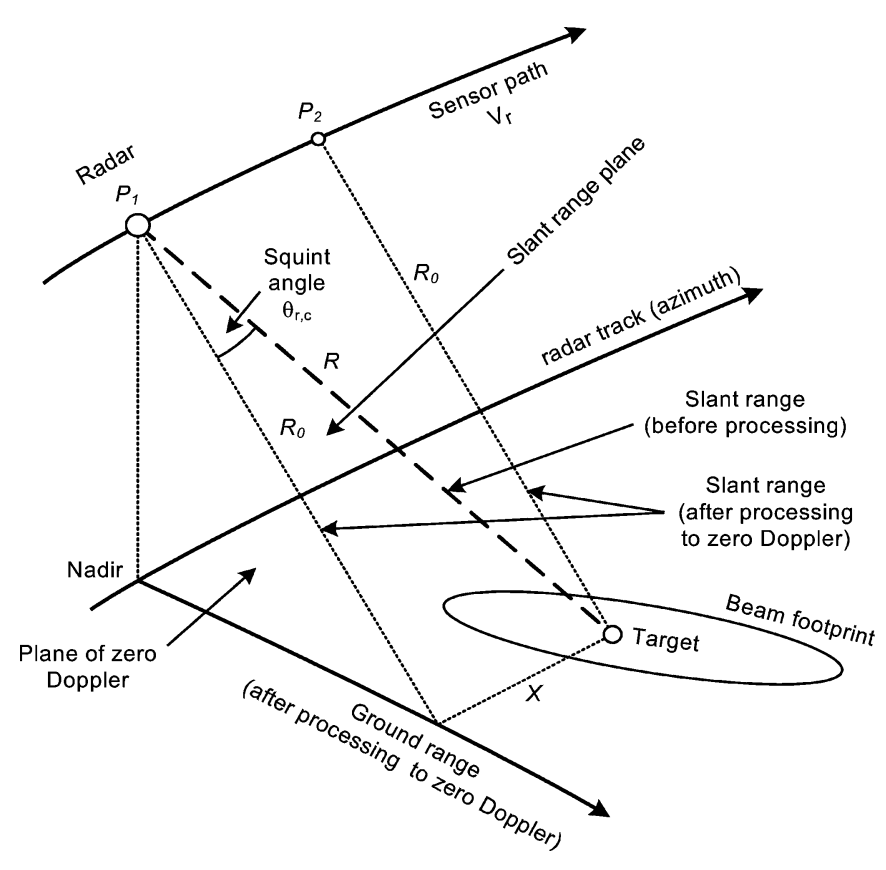

Fig. 1. Geometry of SAR data acquisition in the slant range plane.

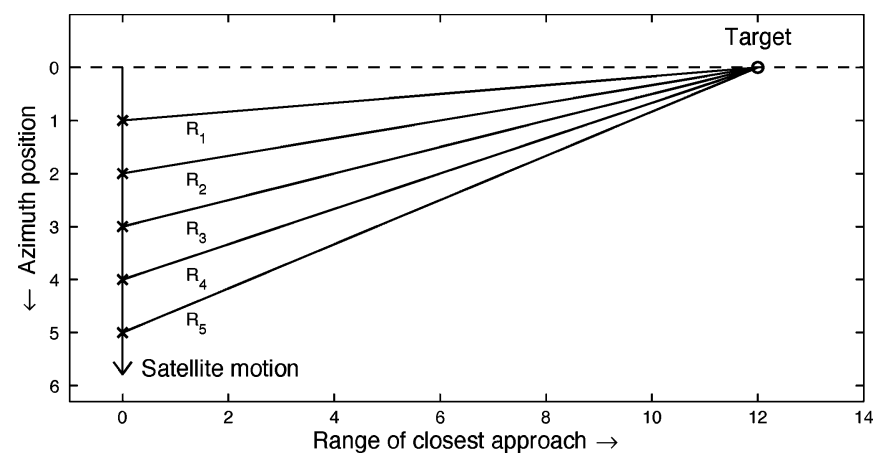

Fig. 2. How slant range to the target varies as the satellite passes by.

Doppler centroid can be found. Note that the conversion factor from slope to Doppler frequency in (1) varies with range, because the orientation of the slant range plane varies with beam elevation angle and $V_{r}$ is mildly range dependent.

Fig. 3 shows the slant range to the target at the five times used in Fig. 2. It is seen that the signal energy can be spread over several range cells during the exposure time. ${ }^{1}$ The variation of range with time is called range cell migration (RCM). The direction of the beam centerline is perpendicular to the locus of target energy at the middle of the target exposure, $\eta_{c}=3$, so the squint angle, $\theta_{r, c}$, is equal to the angle of the linear component of RCM.

As a simple way to illustrate how the centroid can be estimated from received data, the point target response after range compression is examined. The sensing geometry is illustrated in Fig. 2 in the slant range plane, where five positions of the satellite are shown, centered on the time, $\eta=\eta_{c}$, that the beam center crosses the target. The range from the satellite to the target,

\footnotetext{
${ }^{1}$ The units of range and azimuth are arbitrary in this illustration, but the aspect
} ratio is correct.

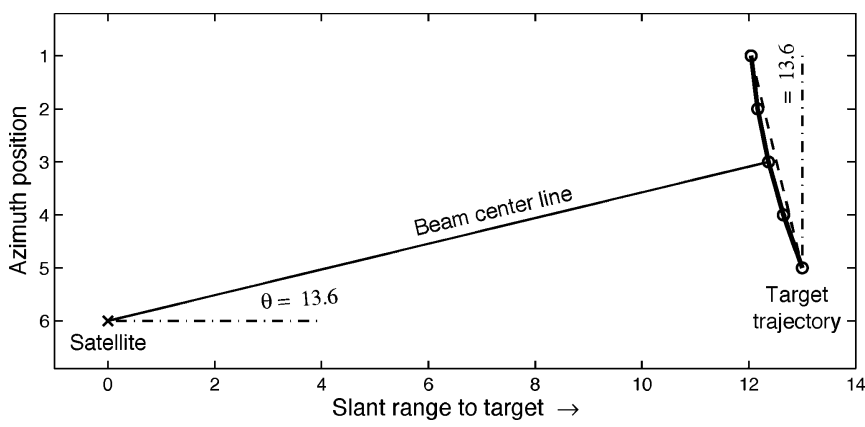

Fig. 3. Range migration of a target as a function of squint angle measured in the slant range plane.

$R(\eta)$, is assumed to be a hyperbola, but can be approximated by the parabola ${ }^{2}$

$$
R(\eta) \approx R\left(\eta_{c}\right)-V_{r} \sin \theta_{r, c}\left(\eta-\eta_{c}\right)+\frac{V_{r}^{2} \cos ^{2} \theta_{r, c}}{2 R\left(\eta_{c}\right)}\left(\eta-\eta_{c}\right)^{2}
$$

From (2), we see that the average RCM slope can be expressed as $V_{r} \sin \theta_{r, c}$ in units of meters per second. From Fig. 3, it is seen that the beam squint angle is also equal to the arc tangent of the slope expressed in units of range meters per azimuth meter. If the slope is positive, that is, the range increases with azimuth time, the antenna has a "backward" or negative squint angle - the Doppler frequency is negative, as is typical of ascending orbits without yaw steering. On the other hand, a negative slope corresponds to a forward squint angle of the antenna.

Therefore, if the RCM slope is measured correctly, the absolute Doppler centroid can be derived directly from (1), with the knowledge of the radar wavelength, $\lambda$, and the geometry variable, $V_{r}$. While there are more accurate ways of estimating the baseband Doppler centroid, the method of measuring the RCM slope can provide a reliable estimate of the Doppler ambiguity number.

\section{RADON TRANSFORM FOR LINEAR FEATURE DETECTION}

In order to extract the information of RCM slope from the range-compressed image, certain image processing techniques can be applied. The Radon transform is an effective technique in extracting the parameters of linear features, such as their slope, even in the presence of noise [6]. Because of its advantageous property in detecting lines with arbitrary orientation, the Radon transform has been successfully used in SAR image processing, such as ship wake detection [8]. This transform integrates intensity along every possible direction in the image and maps this information into a feature space parameterized by the angle with respect to the positive $y$ axis, $\theta$, and the distance from the origin, $\rho$.

The $(\theta, \rho)$ pair form the coordinates of the transformed representation of a line. The concept is that a concentrated point in the transform space represents a linear feature in the image. This approach is particularly suited for noisy images, since the integration process tends to average out intensity fluctuations due to

${ }^{2}$ It is assumed that the satellite and the target remain in the same slant range plane for the exposure time of the target. This plane is unique to a set of targets that share the same slant range of closest approach - the elevation angle of the plane varies with the slant range of closest approach of different targets. 


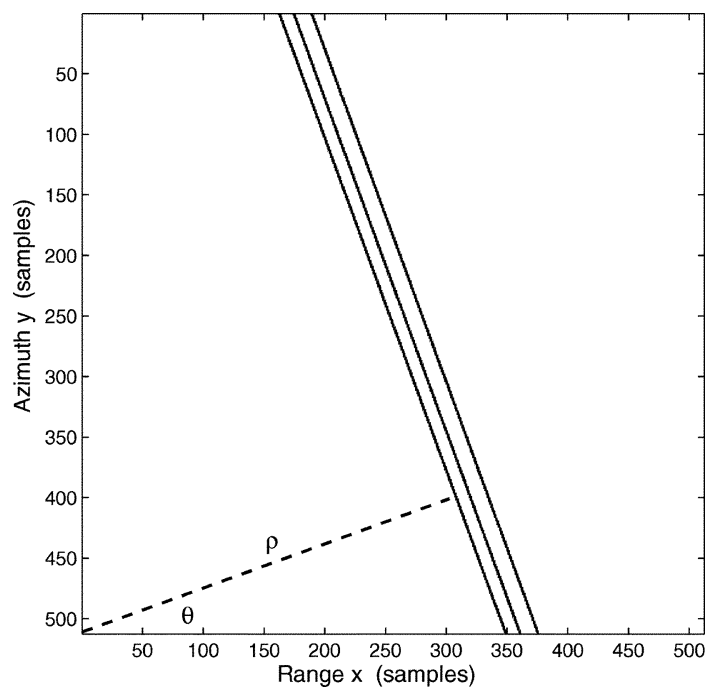

Fig. 4. Simulated SAR magnitude image with three point targets.

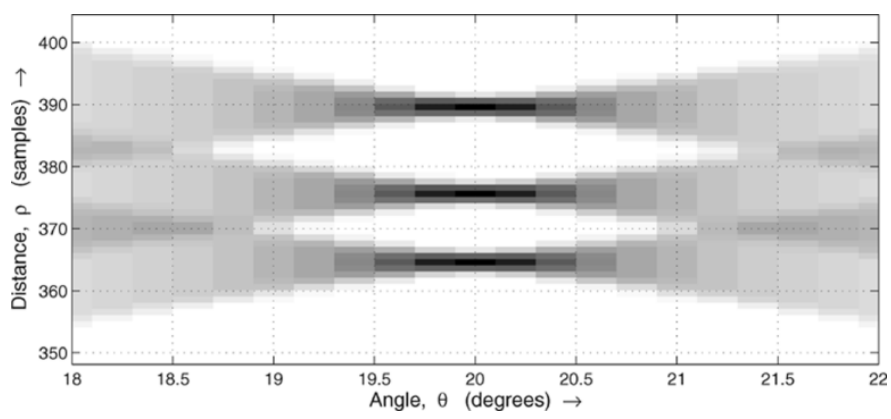

Fig. 5. Radon transform of the SAR image of Fig. 4.

noise. The transform equation for the image, $g(x, y)$, is defined as [6]

$$
\hat{g}(\rho, \theta)=\int_{-\infty}^{\infty} \int_{-\infty}^{\infty} g(x, y) \delta(\rho-x \cos \theta-y \sin \theta) d x d y
$$

where $\delta$ is the Dirac delta function and the factor, $\delta(\rho-x \cos \theta-$ $y \sin \theta$ ), directs the integration along the angle, $\theta$. The range of the integration angle is limited to $0 \leq \theta<\pi$.

To illustrate the relationship between the image coordinates, $(x, y)$, and the transform parameters, $(\theta, \rho)$, a range-compressed SAR magnitude image with three point targets is simulated in Fig. 4. A significant linear RCM is assumed, but there is negligible quadratic RCM. The slope of the target trajectories is $20^{\circ}$, and the Radon transform is taken over angles from $18^{\circ}$ to $22^{\circ}$, in steps of $0.2^{\circ}$.

The result is shown in Fig. 5, where only the central parts of the $\rho$ and $\theta$ axes are displayed. When the integration (3) is taken along the true direction of the lines, the energy is most concentrated along the $\rho$ axis. We see that there are three concentrated areas of energy in the vicinity of $\theta=20^{\circ}$, which indicates the angle of the skewed lines in the image.

In order to quantify the results in more detail, we take vertical slices along the $\rho$ axis of Fig. 5 at several angles. Five slices are shown in Fig. 6, using angles from $19.2^{\circ}$ to $20.8^{\circ}$. It can be seen that the Radon transform result is highly concentrated at the actual skew angle of the target trajectories and increasingly

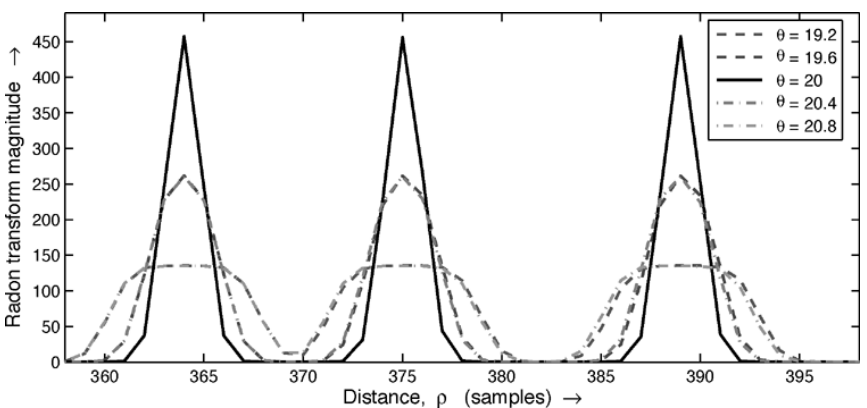

Fig. 6. Vertical slices through the Radon transform of Fig. 5.

dispersed at other angles. In this way, the slope of the lines in the original image can be estimated by finding the angle, $\hat{\theta}$, that gives the maximum concentration of the Radon transform energy along the $\rho$ axis. A "feature space line detector" was proposed in [8], where it was shown that the calculation of the variance of the slices along the $\rho$ direction is a good measurement of the energy concentration.

\section{DOPPLER AMBIGUITY RESOlVER USING THE RADON TRANSFORM}

In this section, we apply Kong's geometry Doppler estimator (GDE) based on the Radon transform to RADARSAT data. We then propose some improvements to the Radon approach arising from satellite SAR processing experience.

\section{A. Applying the GDE to Satellite SAR Data}

Due to its ability of detecting linear features in an image, Kong et al. applied the Radon transform to the Doppler centroid estimation of airborne SAR data [7]. However, due to the lack of bright isolated targets in most SAR data, the measurements are usually not precise enough for the estimation of the baseband part of the Doppler centroid. Since there are several algorithms that can obtain very accurate baseband centroid estimates (especially in areas of very low contrast), we recommend that the Radon transform only be used to obtain the Doppler ambiguity number. The details of the Radon estimator can be stated as follows.

First, take the magnitude or power of the range-compressed image and then calculate the Radon transform. As the Radon transform requires a fair amount of computing time, restrict the angles to within a small range around the expected value. For example, we can estimate the squint angle limits from the geometry model of the satellite SAR system, with the assumption of the maximum yaw/pitch angle deviations. Otherwise, if the range of angles is not easy to estimate a priori, the Radon transform can be applied first using coarse angle increments, and later with a reduced range of angles as the estimates are refined. In the implementation, the Radon transform is calculated with discrete parameter steps, and the transformed image can be expressed as

$R(n, m)=\hat{g}\left(\rho_{0}+n \Delta \rho, \theta_{0}+m \Delta \theta\right), 0 \leq n<N, 0 \leq m<M$

where $\rho_{0}$ and $\theta_{0}$ are the starting values and $\Delta \rho$ and $\Delta \theta$ are the step sizes of the Radon parameters. 


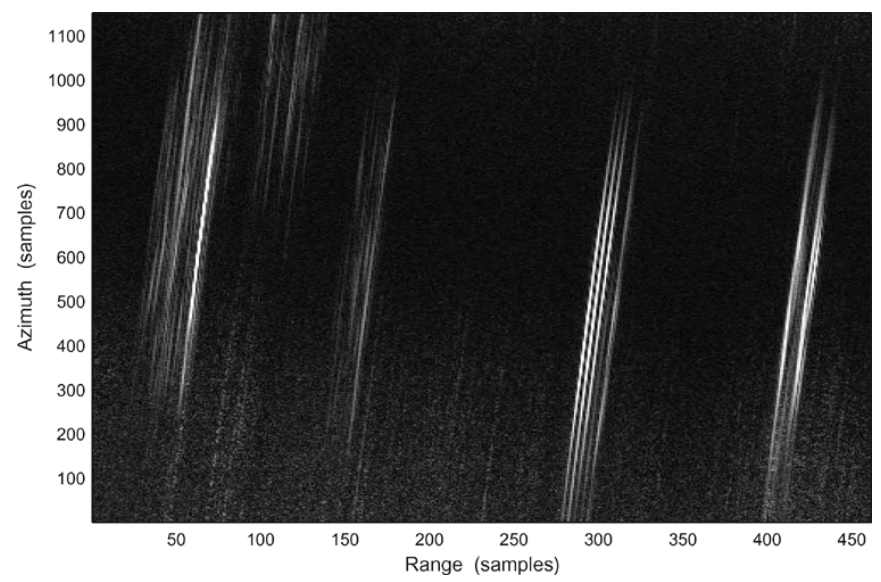

Fig. 7. Example of bright discrete targets (ships) in range-compressed RADARSAT data.

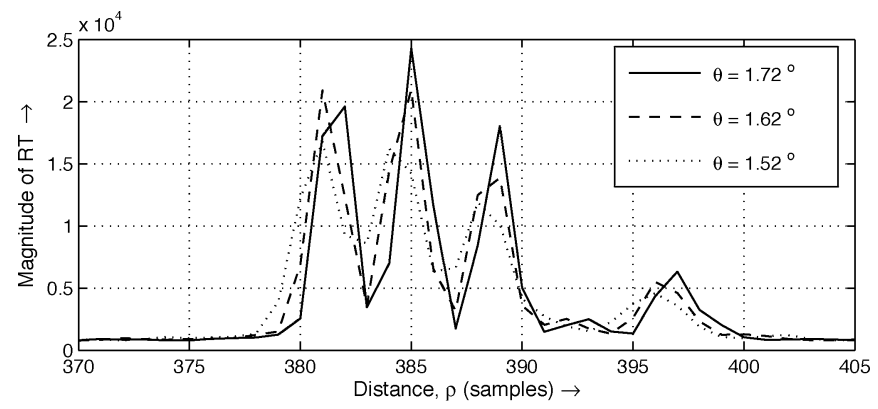

Fig. 8. Slices taken from the Radon transform of the "ships" scene of Fig. 7, taken at different angles.

In order to illustrate the principle of the estimator, we first examine a part of an image with bright discrete targets. The ships in the RADARSAT-1 Vancouver scene provide a good example (see [1, Fig. 12.7]). The ships appear as several near-linear trajectories in the range-compressed magnitude image in Fig. 7. The linear component of range migration is clearly seen. The quadratic term is relatively small in C-band satellite data - about half a range cell.

The Radon transform is applied to the range-compressed image of the "ships" scene of Fig. 7, using angles, $\theta$, from $1.4^{\circ}$ to $2.0^{\circ}$ with an increment of $0.02^{\circ}$. Similar to Fig. 6, Fig. 8 shows three vertical slices through the Radon transform of the scene at different angles (for clarity, the horizontal axis of the figure is expanded so that only one of the ships is shown). It can be seen that the curve at $1.72^{\circ}$ is more concentrated than the curves at the other two angles. By examining a wider range of angles, it was found that the concentration of energy dispersed for angles away from $1.72^{\circ}$, so this value is very close to the true squint angle.

To get better estimation sensitivity, Kong et al. calculate the differential of the transform slices along $\rho$ to emphasize the energy concentration [7]. The results for the three slices of Fig. 8 are shown in Fig. 9. It can be seen that the curve close to the true skew angle exhibits higher variance, while the curves away from the true skew angle have lower variance, as the energy in the integral is more dispersed. In both Figs. 8 and 9 , the slices at $1.82^{\circ}$ and $1.92^{\circ}$ are similar to the slices taken at $1.62^{\circ}$ and $1.52^{\circ}$.

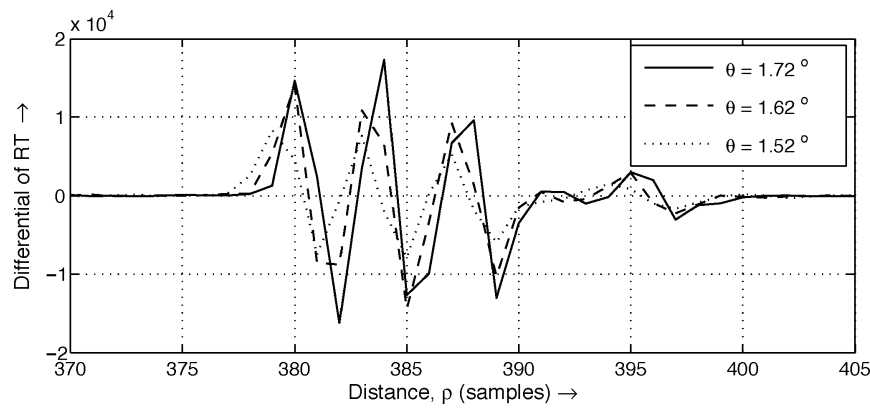

Fig. 9. Differential of the slices of the Radon transform of the "ships" scene, Fig. 8, taken at different angles.

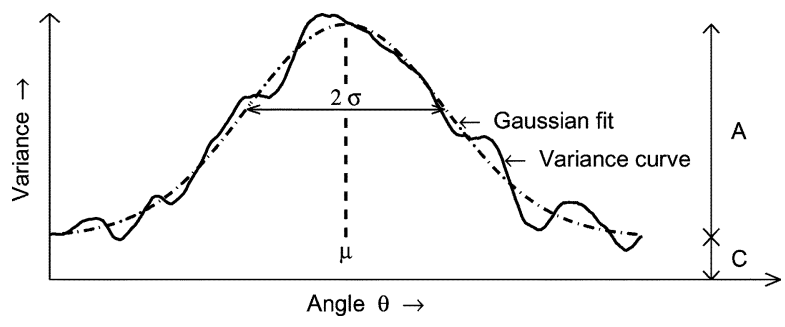

Fig. 10. Fitting a Gaussian model to the variance curve.

To quantify the variability of the differential curve of Fig. 9, the variance of the differential is calculated over the $\rho$ dimension, for each angle in the Radon transform. Following [7], the calculation for the slice at $\theta=m$ can be expressed as

$$
\begin{aligned}
d_{(n, m)} & =R_{(n+1, m)}-R_{(n, m)} \\
\operatorname{Var}_{m} & =\frac{1}{N-1} \sum_{n=0}^{N-2} d_{(n, m)}^{2}-\left\{\frac{1}{N-1} \sum_{n=0}^{N-2} d_{(n, m)}\right\}^{2}
\end{aligned}
$$

where $n$ is the index of $\rho, m$ is the index of $\theta$, and $d_{(n, m)}$ is the differential of the Radon transform, $R_{(n, m)}$, along the $\rho$ axis. The variance curve will have a peak at the angle where the concentration of energy is the greatest.

1) Gaussian Fit to the Variance Curve: In practice, a SAR scene cannot be relied upon to have isolated point targets, and the presence of noise and clutter can distort the variance curve. Rather than simply finding the peak of the variance curve, a curve-fitting approach can find the central angle more accurately. Kong et al. have recommended using a Gaussian function with four unknown parameters [7]

$$
G(x)=A \exp \left\{-\frac{(x-\mu)^{2}}{2 \sigma^{2}}\right\}+C
$$

where $x$ is the independent angle variable and there are four unknown parameters, the amplitude, $A$, the mean or peak location parameter, $\mu$, the standard deviation, $\sigma$, and a pedestal, $C$. A Gaussian curve fit is illustrated by the dashed-dotted line in Fig. 10.

For the "ships" scene of Fig. 7, we estimated the four unknown parameters of the Gaussian fit using MATLAB's fminsearch routine, which uses the Nelder-Mead parameter search procedure [9]. The variance curve and the Gaussian fit are shown in Fig. 11. The peak of the Gaussian fit is located 


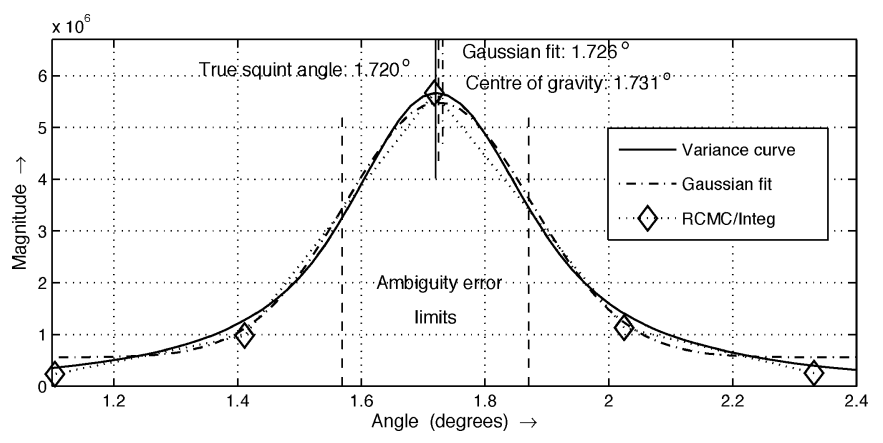

Fig. 11 .Estimating the squint angle by fitting a Gaussian curve to the variance of the differential-the "ships" scene.

at angle $\theta=1.726^{\circ}$, which is very close to the true squint angle of $1.720^{\circ}$. Because the variance curve is quite symmetrical and the noise level is low in this simple case, the Gaussian fit gives almost the same answer as the peak of the sampled curve and the center of gravity (see Section III-B6). However, with more general scene content, the variance curve will be more random, and the Gaussian curve fit will give a more accurate estimate of the central angle.

The closeness of the fit shows that the Gaussian function is an appropriate fitting function to use for this radar data. The diamonds in the figure give the results of the RCMC/integration method, to be discussed in Section IV.

\section{B. Improvements to the $G D E$}

The method described so far in Section III is the one described by Kong [7]. All we have added is an example with satellite data. In this subsection, we discuss some improvements that can be made to Kong's algorithm.

1) Integer Estimation Problem: As in other DAR algorithms, the baseband Doppler centroid should be measured first using the "spectral fit" or "ACCC" algorithms [1]. Then, the baseband Doppler centroid is unwrapped and subtracted from the estimated absolute Doppler frequency, and the result is divided by the PRF. After this, the ambiguity estimate is obtained by a rounding operation. This reduces the ambiguity estimate to the more reliable estimate of an integer (the unwrapping serves to make the ambiguity number the same over the whole scene). The procedure can be expressed as

$$
M_{\mathrm{amb}}=\operatorname{round}\left(\frac{f_{\eta_{c}}-f_{\eta_{c}}^{\prime}}{\mathrm{PRF}}\right)
$$

where $f_{\eta_{c}}$ is the absolute Doppler frequency estimate from the Radon algorithm, $f_{\eta_{c}}^{\prime}$ is the accurate baseband Doppler centroid estimate, and $M_{\mathrm{amb}}$ is the estimated ambiguity number.

2) Removing the Quadratic RCM: As shown in (2), the RCM is not a purely linear function of azimuth time. The RCM has a quadratic component

$$
\Delta R_{\text {quad }}(\eta)=\frac{V_{r}^{2} \cos ^{2} \theta_{r, c}}{2 R\left(\eta_{c}\right)}\left(\eta-\eta_{c}\right)^{2} \approx \frac{V_{r}^{2}}{2 R\left(\eta_{c}\right)}\left(\eta-\eta_{c}\right)^{2}
$$

meters, which can be significant compared to the range cell size. The variable $\eta_{c}$ is the beam center crossing time and $R\left(\eta_{c}\right)$ is the slant range at the time when the target is illuminated by the beam center.

The quadratic component imparts a slope variation along the target trajectory. If the extent of the trajectory corresponding to one PRF is considered, the slope at the ends of the trajectory is the equivalent of \pm 0.5 of an ambiguity compared to the middle of the trajectory. This variation of slope has the effect of broadening the variance function, which reduces the sensitivity of the ambiguity estimate. Therefore, it is recommended to remove the quadratic part from the RCM before taking the Radon transform, to adjust the RCM to a straight line. Removing the quadratic component has the additional advantage of reducing the sensitivity of the estimator to strong partially exposed targets.

The quadratic component of RCM cannot be ignored in some SAR systems. Luckily, for C-Band satellite SAR systems, such as RADARSAT-1 and ENVISAT, the quadratic part of RCM is relatively small. For example, in the "ships" scene that is acquired by the F2 beam of RADARSAT-1, the quadratic RCM is approximately $3 \mathrm{~m}$, about half a range cell. Removing the quadratic RCM would not lead to a significant improvement in this case. However, for L-band satellites, the quadratic part of RCM is about $35 \mathrm{~m}$, and removing it will improve the estimator considerably. Note that the quadratic component of RCM can only be efficiently removed in the azimuth frequency domain and that the Radon method can be adapted to operate in this domain.

An additional point is that the linear component of RCM varies with range, which also broadens the variance curve. This effect can be alleviated by estimating the ambiguity number in smaller range extents, as done in the spatial diversity approach.

3) Localized Radon Transform: If a part of an image can be identified that has strong discrete targets, the estimator will work better if the Radon transform is restricted to that region, as we did in the "ships" scene. This is referred to as the localized Radon transform in [6]. If the correct ambiguity can be found from only a small part of a scene, the result can be applied to the whole scene, as long as the baseband centroid is unwrapped correctly.

4) Secondary Range Compression: Depending upon the radar system parameters and the squint angle, secondary range compression (SRC) may have to be applied to sharpen the focus in the range Doppler domain. As discussed in [1, ch. 6], without SRC the range-compressed image can be defocused in the azimuth frequency domain, even though it is well focused in the time domain. On the other hand, if SRC is applied with the range compression filter, the image is well focused in the azimuth frequency domain, but possibly not in the time domain.

Therefore, if we apply the Radon transform to RCM slope detection in the time domain (as we do in the examples in this paper), SRC should be implemented after the estimator. Otherwise, if the slope is detected in the frequency domain, SRC should be implemented before the estimator.

5) Estimator Quality Criteria: In order to avoid corruption by bad estimates from the areas with very weak backscatter or low contrast, quality criteria can be used to detect and remove bad estimates from the final Radon estimate, when the "spatial diversity" approach [10] is used. In addition to the data quality criteria of SNR and contrast used in [10], the following four 


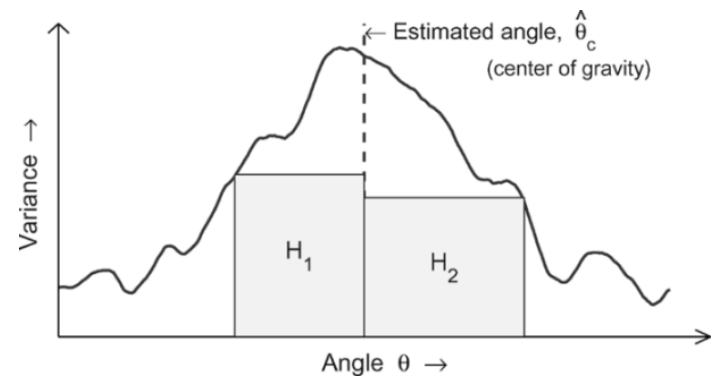

Fig. 12. Finding the "peak" of the variance curve by the "center of gravity" method.

estimator quality criteria that are specific to the operation of the Radon method were examined.

a) Gaussian Fit Flag: If the search for the four Gaussian fit parameters does not converge or the fit parameters are beyond a reasonable range, we declare that the fit procedure fails (flag $=0$ ). If the fit is deemed successful, the flag is set to 1 and the next three criteria are examined.

b) Fit Distortion: The Fit Distortion is defined as the normalized standard deviation of the difference between the measured variance curve and the Gaussian function.

c) Peak to Pedestal Ratio (PPR): In addition, the shape of the fitted Gaussian function can be used to measure how good the estimate is. The PPR is obtained from the Gaussian fitting parameters by PPR $=(A+C) / C$. The bigger the ratio, the better the fit.

d) Width of Gaussian Fit: This width is obtained from the $\sigma$ parameter of the Gaussian fit. On the assumption that "sharper" variance functions indicate better estimates, lower values of $\sigma$ are preferred.

6) "Center of Gravity" Method: If you want to avoid the complexity of curve fitting, a simpler and almost as accurate method of finding the location of the peak of the variance curve is to find the "center of gravity" of the curve. The central angle is found by this method using

$$
\hat{\theta}_{c}=\frac{\sum_{n=1}^{N} \theta_{n} \operatorname{Var}_{n}}{\sum_{n=1}^{N} \operatorname{Var}_{n}}
$$

where $\operatorname{Var}_{n}$ is the variance value at the angle, $\theta_{n}$. As illustrated in Fig. 12, this method is equivalent to finding the angle, $\hat{\theta}_{c}$, that balances the integral of the shaded areas, $H_{1}$ and $H_{2}$, when the integral is taken to the left and to the right of the estimated angle. When the variance function is not symmetric because of noise and clutter, the center of gravity is a more accurate estimate than simply the position of the maximum value.

The result of the center of gravity method applied to the "ships" scene is shown in Fig. 11. While the Gaussian fitting method obtains the best estimate in this case, the error of the center of gravity method is fairly small, well within the ambiguity error limit (the ambiguity error limit represents the range of angles that do not lead to a Doppler ambiguity error). Therefore, the center of gravity can be viewed as a simpler measurement of the squint angle than the Gaussian fit, and may be adequate for many applications. Further comparisons are given in Section V.

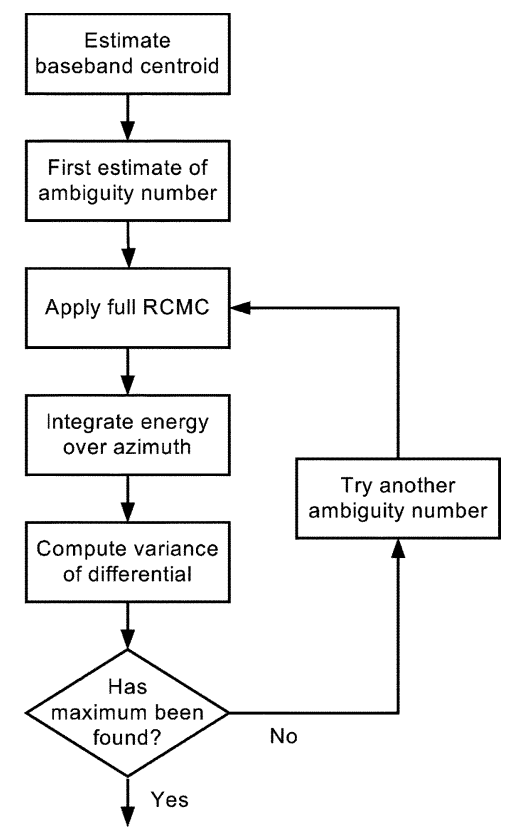

Fig. 13. Flowchart of the RCMC/integration method of finding the Doppler ambiguity.

\section{RCMC/INTEGRATION METHOD}

A new method of Doppler ambiguity resolution can be defined, as suggsted by the following points.

- Because the baseband centroid can be found accurately, the search for the correct Doppler ambiguity can be confined to integer numbers (i.e., the ambiguity number, $M_{\mathrm{amb}}$ - see Section III-B1).

- While the Radon transform is computed for a fine grid of angles (as in Section III), only a few discrete angles need be searched for the integer numbers.

- The estimation of linear features is more sensitive once the quadratic RCMC is performed.

- RCMC has to be done for the processing anyway, so doing it at the estimation stage is not a significant burden.

The method that is suggested by these considerations is an iterative or search scheme outlined in Fig. 13. It starts from rangecompressed, SRCed data in the azimuth frequency domain, and operates as follows.

1) Find the baseband centroid as a function of range over the whole scene and unwrap over PRF jumps.

2) Make a preliminary estimate of the ambiguity number from geometry, including its likely range of values (consider the possible angles of the radar beam, and include the Earth rotation component).

3) Apply full RCMC (e.g., both the linear and quadratic parts) over the whole scene, using the candidate ambiguity number.

4) Convert the radar data to magnitude or power units and integrate the energy over the azimuth axis to obtain a curve of energy versus range.

5) Compute the variance of the differential of the integrated energy over the range variable, as done in the Radon method. 


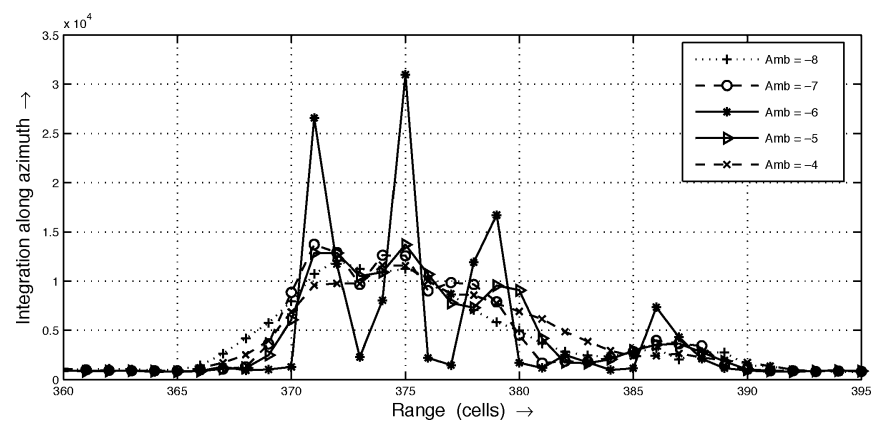

Fig. 14. Integration along azimuth of the "ships" scene after RCMC_-for each curve, RCMC is done with a different assumed ambiguity number.

6) Check whether the variance has gone through a maximum as a function of ambiguity number.

7) If a maximum is reached, the correct ambiguity has been found. If not, increment the ambiguity number, and repeat from Step 3). Search for the correct ambiguity number in both directions.

\section{A. Discussion and Example}

Essentially, this method replaces the Radon transform with a simple integration of the image energy over one dimension (azimuth). The RCMC removes the quadratic component and variation of slope with range discussed in Section III-B2. Only one ambiguity number results in RCMCed data that are aligned accurately in azimuth, and quality checks are an effective way of checking the accuracy of the alignment.

The baseband centroid estimates must be unwrapped so that a single ambiguity number applies over the whole scene. The spatial diversity, curve fitting method is the most reliable way of ensuring accurate estimates that vary smoothly over the scene, and that the unwrapping is correct [10].

The RCMC is best applied in the azimuth frequency domain, as in the range Doppler algorithm, so that the quadratic RCMC can be performed efficiently. The subsequent estimation can be done in this domain, which is why SRC should be applied with the range compression filter (i.e., using Option 3 described in $[1$, ch. 6]).

Note that the baseband estimates are used in different ways in the two approaches. In the RCMC/integration method, they are used to determine the curve for the RCM correction - where a wrong baseband estimate would increase the ambiguity level. They are also used (after unwrapping) to reduce the estimation of the ambiguity to the more reliable estimate of an integer. In the Radon method, the baseband estimate is only used at the end to make the estimate near an integer in (8).

As in any ambiguity estimation method, parts of each scene will likely yield bad estimates. These usually occur in areas of low image SNR and/or low image contrast. Using the spatial diversity approach over small blocks of the scene, quality criteria can be used to reject the bad blocks and obtain higher confidence in the answer. In addition to measuring the SNR and contrast of each block, the peak-to-mean ratio of the curve of variance versus ambiguity number is a suitable quality parameter.

The RCMC/integration method was applied to the ships of Fig. 7. The results of the azimuth integration using several ambiguity numbers are shown in Fig. 14. It can be seen that the result with the highest variance is obtained when the correct ambiguity number, $M_{\mathrm{amb}}=-6$, is used.

The correct answer is even more apparent when the differential and variance are taken over the range variable. These results are plotted in Fig. 11 with the $\diamond$ symbols, which shows that the RCMC/integration results agree closely with the Radon transform results.

\section{EXPERIMENTS WITH RADARSAT-1 DATA}

As shown in the results of the "ships" scene, the estimators using the Radon transform and the RCMC/integration method work well in an area with isolated bright targets, since the targets have clearly defined linear features after range compression. We now examine how the estimators behave with a more general scene content.

In order to test their performance on different kinds of terrain in satellite SAR data, the RADARSAT-1 Vancouver scene is selected, as it contains areas of salt water, fresh water, city, suburbs, farmland, forest, and mountains. The salt water is in the Gulf of Georgia - it has a relatively low surface roughness as it is only $35 \mathrm{~km}$ wide and is sheltered from the open ocean.

As described in [10], we use the "spatial diversity" approach, where the scene is divided into blocks containing different terrain types. As only one ambiguity number has to be estimated over the whole scene, areas that lead to bad estimates can be removed, and an average or "majority vote" can be taken over the remaining blocks. In these experiments, we divide the whole Vancouver scene into 12 (range) $\times 19$ (azimuth) blocks, each with 655 range cells and 1024 lines. The block borders are outlined in the range-compressed "image" in Fig. 12.15 of [1].

After range compression, the accurate "spectral fit" baseband Doppler estimator is applied and the PRF wraparound is removed. The quadratic component of RCM is removed. Then, the Radon and the RCMC/integration methods are applied to estimate the Doppler ambiguity. The quality criteria are measured for each block to test their effectiveness and to remove biased or noisy estimates.

\section{A. Format of the Experimental Results}

Typical experimental results are shown in Figs. 15-18. Each figure shows the results of the 12 blocks in one particular row of the scene. The row number refers to one of the numbers 1-19 annotated along the vertical axis of Fig. 12.15 in [1] (the annotation is adjacent to the upper boundary of the corresponding row). There are 12 blocks in each row, corresponding to the numbers 1-12 on the horizontal axis of Fig. 12.15 in [1] (the annotation is adjacent to the right boundary of the corresponding block). In Figs. 15-18, the Block 1 results are shown in the top left subplot - the block numbers increase from left to right then from top to bottom, corresponding to increasing range in the scene. The Block 12 results are in the lower right subplot of each figure.

The horizontal axis of each subplot refers to the angle used in the Radon transform, but is expressed in units of ambiguity number for compatibility with the RCMC/integration method and for visibility of the result in ambiguity units. The unwrapped baseband centroid is removed from the estimate, so the answer 

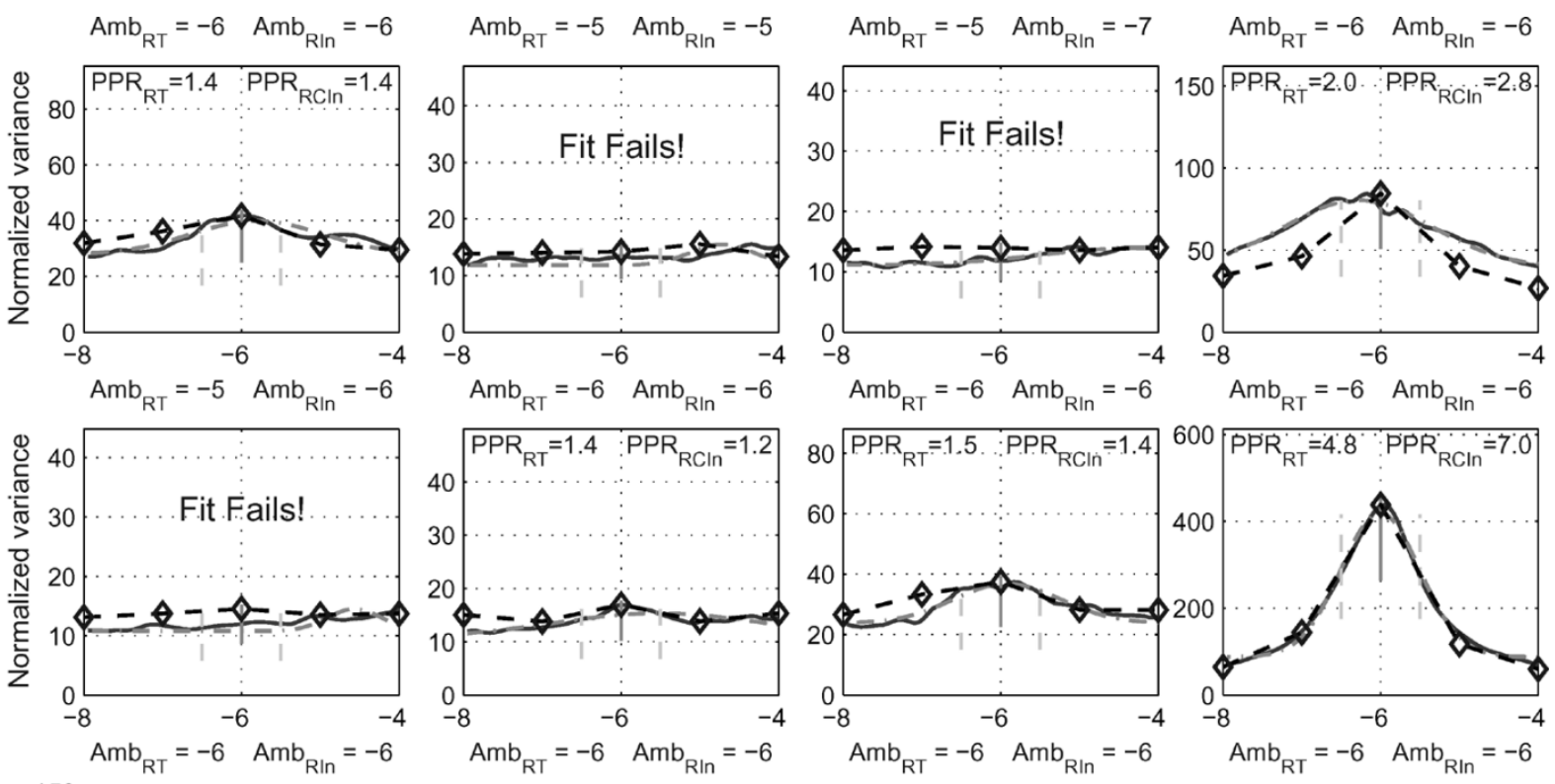

$\mathrm{Amb}_{\mathrm{RT}}=-6 \quad \mathrm{Amb}_{\mathrm{RIn}}=-6$
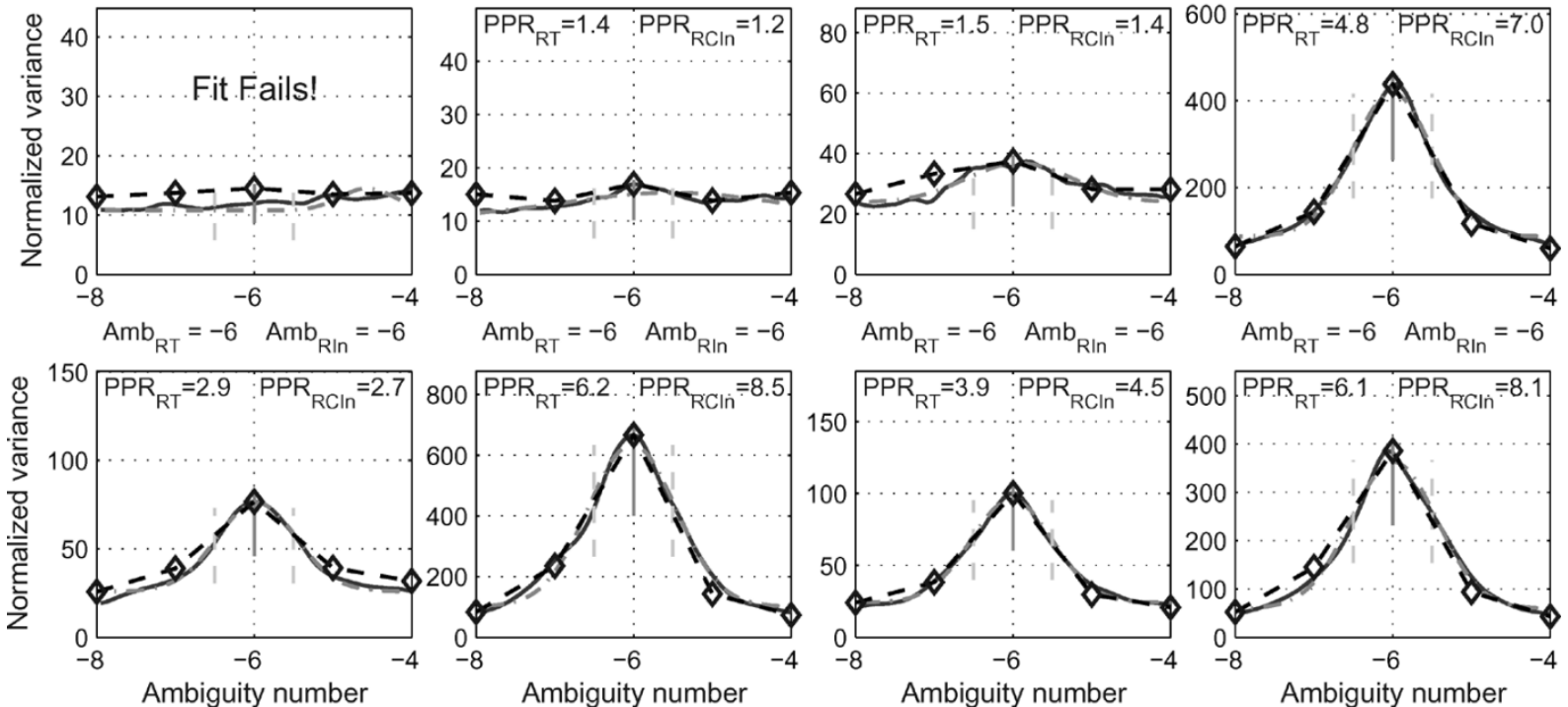

$\mathrm{Amb}_{\mathrm{RT}}=-6 \quad \mathrm{Amb}_{\mathrm{R} \text { In }}=-6$

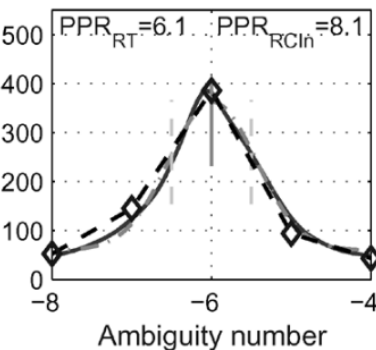

Fig. 15. Estimation results from the Vancouver scene, Row 1.

should be an integer. The correct answer is indicated by the vertical solid line and equals -6 for this scene. The vertical dashed lines indicate the $\pm \mathrm{PRF} / 2$ limits-exceeding these limits results in an ambiguity error.

In each panel, the solid curve shows the measured variance of the difference of the Radon transform, referred to as the "variance curve." Range compression is performed without SRC, and then the Radon transform is applied. The Gaussian fit to the Radon variance is indicated by the dashed-dotted line, and a quality criterion is expressed in the peak-to-pedestal ratio, $\mathrm{PPR}_{\mathrm{RT}}$. The center of gravity and the other quality criteria discussed in Sections III-B5 and III-B6 are also computed, but are not shown on the plots for clarity.

The RCMC/integration results are shown by the diamonds and the connecting dashed lines, using data that has been range-compressed with SRC. These results are more quantized as they are only calculated at integer ambiguity numbers. The peak-to-pedestal ratios, $\mathrm{PPR}_{\mathrm{RCin}}$, are annotated. The meaning of this PPR is analogous to the Radon Gaussian fit PPR, but it cannot be directly compared with it because of the quantization of the calculations. Finally, the estimated ambiguity values are given at the top of each panel for the Radon transform (left) and the RCMC/integration methods (right).

\section{B. Discussion of the Results}

The results of four featured image rows are shown in Figs. 15-18. Refer to Fig. 12.15 in [1] to observe the image content of each block.
1) Image Row 1 (Fig. 15): Block 1 is half on land and half in the water. Even though the land is on a wooded island with few cultural features, there is enough contrast in the land to give the correct estimate with both methods, although the peak to pedestal ratios are quite low compared to other successful blocks. Block 7 is mainly in the water, but has enough land area to give a good result.

Blocks 2, 3, 5, and 6 are almost entirely in the water, with no bright targets and a low SNR because the water is not rough. The curves of variance versus ambiguity number are spread out and rather random, due to the almost total lack of contrast. In three of these cases, the Radon variance curve does not have a well-defined peak and the Gaussian fit fails. In Block 5, the RCMC/integration method just barely gives the correct estimate. Both estimates are correct in Block 6, but the PPR is low, which indicates a higher probability of error.

Block 4 is also in the water, but contains the partial exposure of a single ship. The Radon variance curve and its Gaussian fit have a peak just outside the ambiguity error limit and gives an incorrect result. However, the RCMC/integration method has a well-defined peak at $M_{\mathrm{amb}}=-6$, and gives the correct result. Note that the RCMC/integration method is not affected by partial exposures because the full RCMC removes the azimuth dependence of the target slope (in general, the slope estimation methods are not as upset by partial exposures as methods based on measuring frequency or phase).

In comparison, Blocks 8-12 are in a suburban/agricultural/wooded area of northern Washington state, with some 

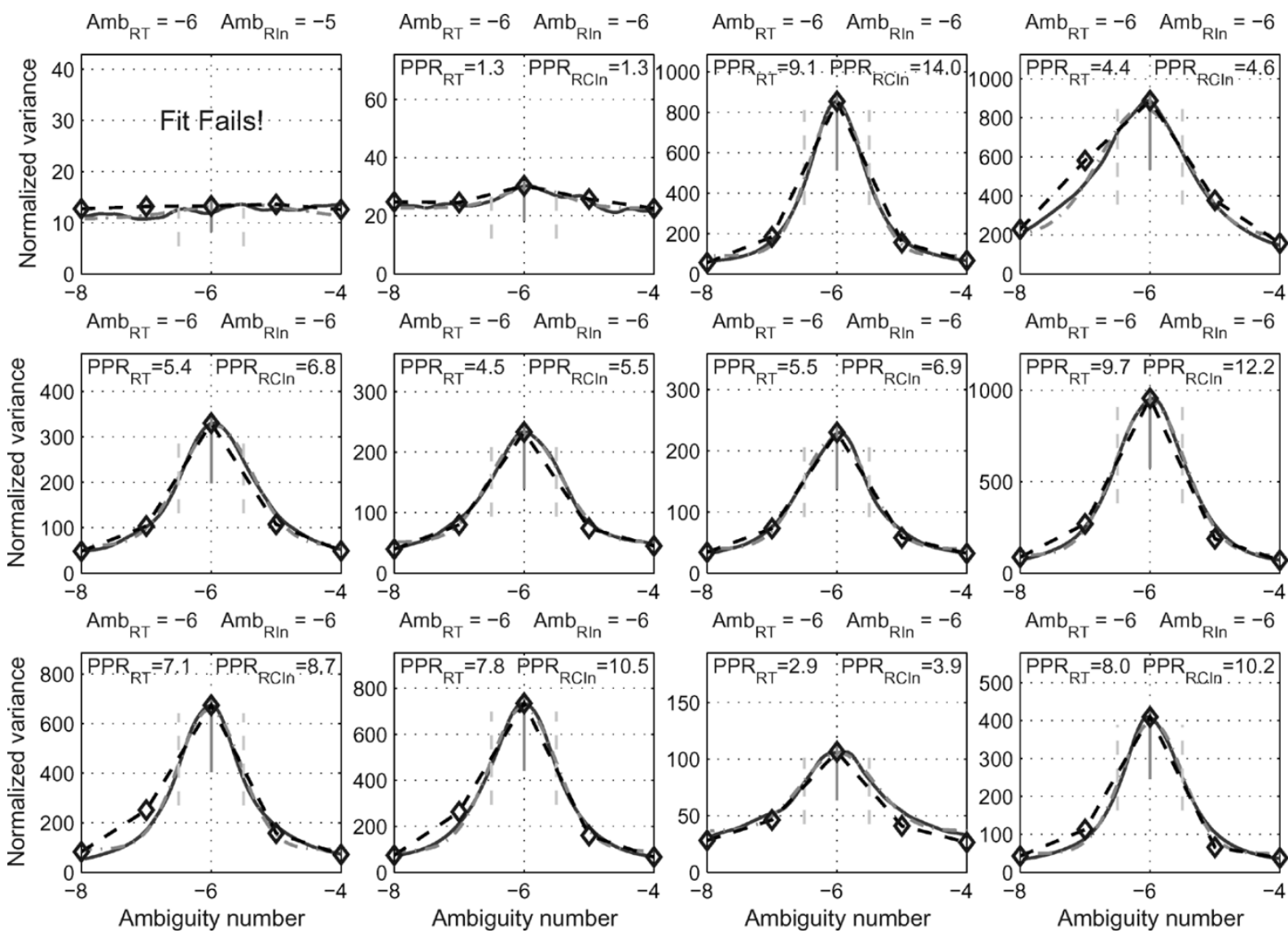

Fig. 16. Estimation results from the Vancouver scene, Row 6.

cultural features but with relatively low contrast. The shapes of the variance curves are sharper, narrower, closer to the Gaussian function and have a larger PPR than the other blocks. As a result, the angle estimates are well within the ambiguity error limits.

The quality criteria are found to reflect the effect of the scene content on the accuracy of the estimates-when the block has fewer bright targets, less contrast or lower SNR, the PPR and the height of the variance curve are smaller. The estimates of the low-SNR Blocks 1-6 have a significant randomness and should be removed from the estimate average by the SNR, PPR, or other quality criteria, but are shown here for discussion purposes.

2) Image Row 6 (Fig. 16): Block 1 and the first half of Block 2 of Row 6 are in the water, and the remaining of the blocks are in a suburban/farmland/forested area south of Vancouver. Block 1 gives a wrong result (as in several of the Row 1 blocks), but Block 2 gives the correct result for both methods. In the case of Block 2, both methods give a clear peak at $M_{\mathrm{amb}}=-6$ even though the peak-to-pedestal ratio is not large.

All the land blocks exhibit well-defined peaks and give correct results. Blocks $8-10$ and 12 contain some strong discrete reflectors which give large PPRs, with correspondingly sharper peaks in the variance curves.

3) Image Row 8 (Fig. 17): Row 8 is included because Block 10 gave a very poor result in the baseband estimator owing to a very strong, partially exposed reflector-see Fig. 12.16(b) in [1]. The Radon result is biased by about 0.2 of a PRF, but is within the error limit, while the RCMC/integration method gives a clear, strong result. The PPRs are lower than in the surrounding blocks.

4) Image Row 15 (Fig. 18): Row 15 covers a heavily forested, mountainous region with elevation differences up to $1700 \mathrm{~m}$. There is some radar layover in this part of the scene. There are no cultural targets, except a few in Block 3. The PPRs are much lower than in the land regions of the other nonmountainous rows. Despite this, only Block 6 experienced an incorrect result with these estimators.

\section{Comparison of Results of Each Method}

In Table I, we compare the performance of the Radon and RCMC/integration estimators over a consistent set of blocks of the Vancouver scene. Each estimator has different quality measures, but in order to compare the estimators fairly, we only use only one quality criterion in this comparison so that the same blocks are rejected for each estimator. The criterion of "SNR > $-1 \mathrm{~dB}$ " is used, and 28 out of the 228 blocks are rejected. These are mainly the blocks that are dominated by water areas.

A convenient way to assess the results is to look at the ambiguity estimate in units of PRFs after the baseband centroid is subtracted but before the rounding in (8) is done. The results should all equal -6 for this scene, but have a random component due to the clutter and noise in the radar data. The second column in Table I gives the mean value of all the blocks, the next column gives the standard deviation, while the final column 

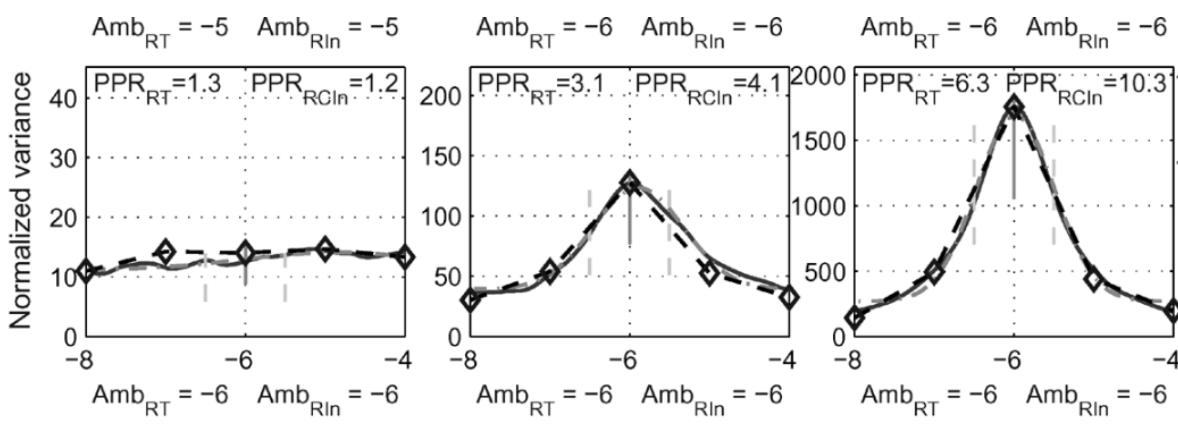

$\mathrm{Amb}_{\mathrm{RT}}=-6 \quad \mathrm{Amb}_{\mathrm{RIn}}=-6$
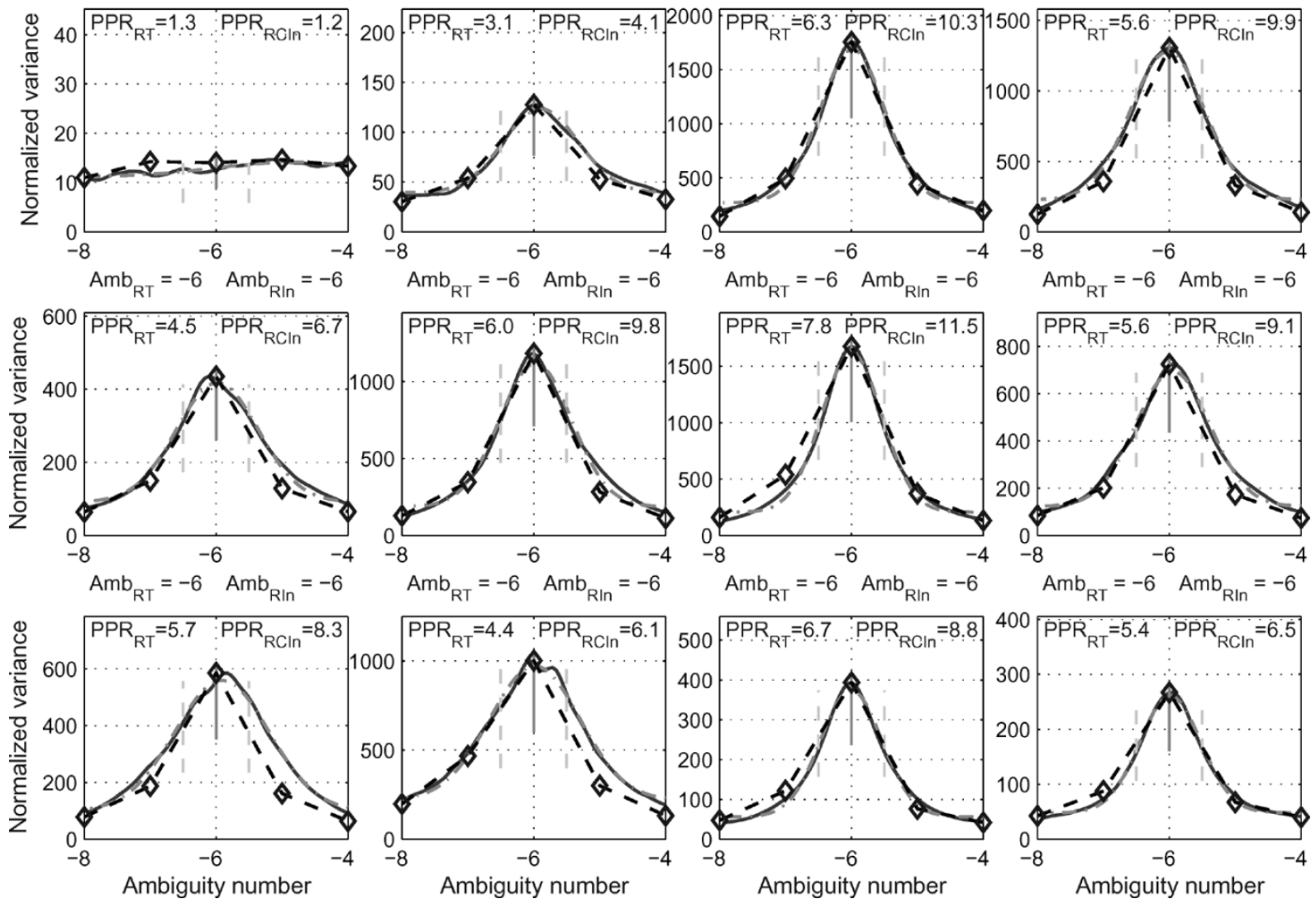

Fig. 17. Estimation results from the Vancouver scene, Row 8.

TABLE I

COMPARISON OF DAR METHODS FOR THE VANCOUVER SCENE

\begin{tabular}{|l|c|c|c|}
\hline & $\begin{array}{l}\text { Mean } \\
(\mathrm{PRFs})\end{array}$ & $\begin{array}{l}\text { St. Dev. } \\
(\mathrm{PRFs})\end{array}$ & $\begin{array}{c}\text { Success } \\
\text { rate (\%) }\end{array}$ \\
\hline Standard MLCC & -5.83 & 1.36 & 41 \\
\hline Standard MLBF & -5.72 & 1.09 & 62 \\
\hline MLBF using ILP & -5.89 & 0.43 & 85 \\
\hline Radon with CofG & -5.97 & 0.12 & 99 \\
\hline Radon with Gaussian & -5.96 & 0.11 & 99 \\
\hline RCMC/integration & -6.01 & 0.10 & 99 \\
\hline
\end{tabular}

gives the percentage of blocks with the correct estimates of the 200 nonrejected blocks.

Other DAR methods are included in the table for comparison. The first two rows assess the standard versions of the original MLCC and MLBF algorithms described in [5]. The next row gives the results of a new version of the MLBF algorithm that uses an improved beat frequency estimator rather than a fast Fourier transform [11]. The last three rows assess the Radon transform method using the center of gravity measurement, the Radon method with the Gaussian fit, and lastly the RCMC/integration method.

The mean values in Column 2 are all as close enough to -6 as not to matter. However, the standard deviation of the Doppler estimates in Column 3 reveals the degree of randomness of each method. The standard versions of the MLCC and MLBF algorithms show a fair amount of variability, as many people have experienced. The improved method of beat frequency estimation in the MLBF algorithm shows considerably less variation.

However, the estimates based on the Radon transform and the RCMC/integration method are clearly giving much better estimates with less variability. The Gaussian fit method of estimating the slope gives better results than the center of gravity method, likely because it uses a more appropriate function in the fitting procedure. Finally, the RCMC/integration method gives equal or better results than the Radon methods and may be the best one of all (note that the standard deviation value for the RCMC/integration method is somewhat affected by the integer-quantized solutions).

1) Assessment of Quality Criteria: In the Vancouver scene results, we see how the estimator can behave differently with different scene content. Estimator quality criteria can be used to automate the assessment of scene content and the estimation results, to determine the suitability of each part of the scene for providing robust ambiguity estimates. Of the four quality measures discussed below, the first two are properties of the scene, while the last two are properties of the estimator and do depend upon the estimation algorithm.

Signal-to-Noise Ratio: Experience has shown that SNR is important to all ambiguity estimation procedures (it is not as important to the baseband estimators). If the receiver noise level can be estimated for an area of the scene, a threshold can be placed a few decibels above to set a rejection criterion. 


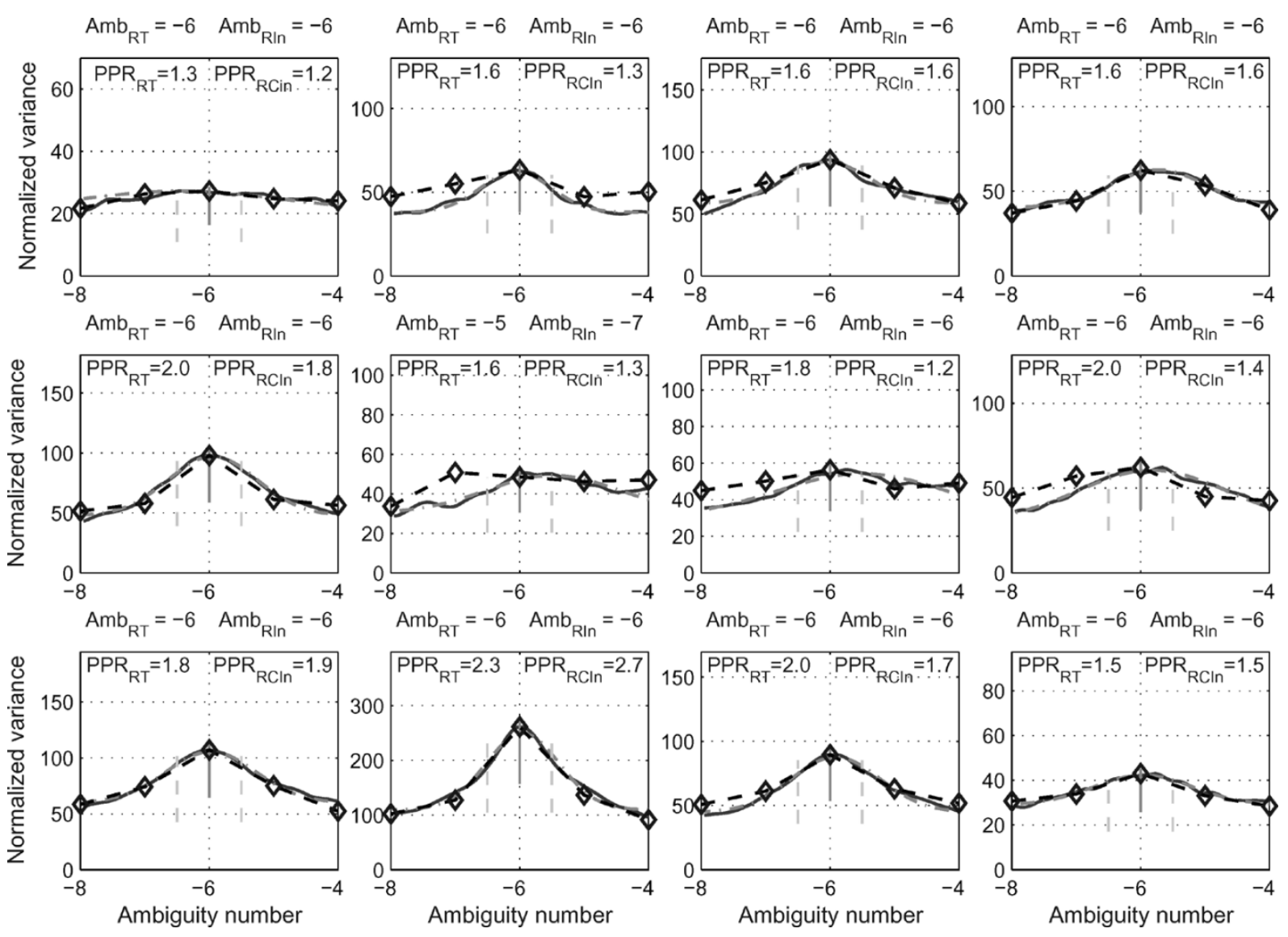

Fig. 18. Estimation results from the Vancouver scene, Row 15.

TABLE II

Performance of the Gaussian Fit Flag as a Quality Measure

\begin{tabular}{|l|c|c|}
\hline & Flag $=1$ & Flag $=0$ \\
\hline Good estimate & 209 & 2 \\
\hline Bad estimate & 5 & 12 \\
\hline
\end{tabular}

Image Contrast: Unlike the WDA and MLCC methods, high image contrast is important to the slope estimation methods. Specifically, the presence of cultural features in the image help these methods, but are not absolutely necessary for good performance.

Quality of Gaussian Fit: Looking at the Gaussian fitting flag for the whole 228 data blocks, 209 blocks gave correct ambiguity estimates when the fit was deemed successful (flag $=1$ ), while only two blocks gave a wrong estimate when the flag $=1$ (see Table II). When the fit was deemed unsuccessful (flag $=0$ ), five blocks were indeed bad estimates, while 12 blocks actually had correct estimates. So, if the fitting flag $=1$ were the only quality criterion used, the correct ambiguity would be obtained if the results were averaged or a "majority vote" were taken. However, it is still recommended to add other quality criteria to the rejection process, such as the SNR, contrast, fit width, fit standard deviation, and PPR.

Peak-to-Pedestal Ratio: The PPR gives a good indication of the sensitivity of the two slope estimation methods. If the contrast in the scene is high enough that linear features are recognizable by the algorithms, the PPR will have a high value, say $>3$. The higher the PPR, the more sensitive the slope measurement is.

\section{CONCLUSION}

Results with simulations and real RADARSAT data show that the estimate of the slope of linear features in a SAR image can be an effective way of resolving the Doppler ambiguity number. The Radon transform method [7] was evaluated and some improvements made, and a new, simpler method based on RCMC and azimuth integration was presented. The slope estimation methods work well in medium to high-contrast scenes, even when no prominent targets are visible. The estimators gave the correct result in almost all areas of the tested RADARSAT scene, except in areas of calm water where the image SNR is very low.

The estimates are made after range compression, and optionally after the azimuth Fourier transform, which are the most convenient places of the processing chain to apply the estimator. Methods are introduced to reduce the effects of slope variation with range and partial azimuth exposures. The RCMC/integration method is not affected at all by these data properties, while the Radon method is only affected a small amount. The results are also improved by subtracting the baseband Doppler centroid and applying SRC when needed.

Quality measures derived from the data and from the estimator results are a useful way of avoiding areas in a scene that do not give reliable estimates. Removing difficult areas gives 
the estimators a high confidence level when a spatial diversity, global fitting approach is taken.

\section{ACKNOWLEDGMENT}

The authors are grateful to the Natural Sciences and Engineering Research Council of Canada for funding for this project, and to MacDonald Dettwiler for the RADARSAT data used in the study.

\section{REFERENCES}

[1] I. G. Cumming and F. H. Wong, Digital Processing of Synthetic Aperture Radar Data Algorithms and Implementation. Norwood, MA: Artech House, 2005.

[2] I. G. Cumming, P. F. Kavanagh, and M. R. Ito, "Resolving the Doppler ambiguity for spaceborne synthetic aperture radar," in Proc. IGARSS, Zurich, Switzerland, Sep. 8-11, 1986, pp. 1639-1643.

[3] C. Y. Chang and J. C. Curlander, "Application of the multiple PRF technique to resolve Doppler centroid estimation ambiguity for spaceborne SAR," IEEE Trans. Geosci. Remote Sens., vol. 30, no. 5, pp. 941-949, Sep. 1992.

[4] R. Bamler and H. Runge, "PRF-ambiguity resolving by wavelength diversity," IEEE Trans. Geosci. Remote Sens., vol. 29, no. 6, pp. 997-1003, Nov. 1991.

[5] F. H. Wong and I. G. Cumming, "A combined SAR Doppler centroid estimation scheme based upon signal phase," IEEE Trans. Geosci. Remote Sens., vol. 34, no. 3, pp. 696-707, May 1996.

[6] A. L. Warrick and P. A. Delaney, "Detection of linear features using a localized Radon transform," in Conf. Rec. 30th Asilomar Confe. Signals, Systems and Computers, vol. 2, Nov. 3-6, 1996, pp. 1245-1249.

[7] Y.-K. Kong, B.-L. Cho, and Y.-S. Kim, "Ambiguity-free Doppler centroid estimation technique for airborne SAR using the Radon transform," IEEE Trans. Geosci. Remote Sens., vol. 43, no. 4, pp. 715-721, Apr. 2005.

[8] A. C. Copeland, G. Ravichandran, and M. M. Trivedi, "Localized Radon transform-based detection of ShipWakes in SAR images," IEEE Trans. Geosci. Remote Sens., vol. 33, no. 1, pp. 35-45, Jan. 1995.

[9] J. A. Nelder and R. Mead, "A simplex method for function minimization," Comput. J., vol. 7, pp. 308-313, 1965.

[10] I. G. Cumming, "A spatially selective approach to Doppler estimation for frame-based satellite SAR processing," IEEE Trans. Geosci. Remote Sens., vol. 42, no. 6, pp. 1135-1148, Jun. 2004.
[11] S. Li and I. G. Cumming, "Improved beat frequency estimation in the MLBF Doppler ambiguity resolver," in Proc. IGARSS, Seoul, Korea, Jul. 25-29, 2005.

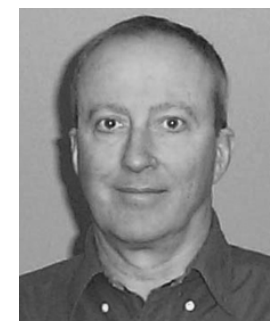

Ian G. Cumming (S'63-M'66-SM'05-LS'06) received the B.Sc. degree in engineering physics from the University of Toronto, Toronto, ON, Canada, in 1961, and the Ph.D. degree in computing and automation from Imperial College, University of London, London, U.K., in 1968.

He joined MacDonald, Dettwiler and Associates, Ltd., Richmond, BC, Canada, in 1977, and since that time, he has developed synthetic aperture radar signal processing algorithms, including Doppler estimation and autofocus. He has been involved in the algorithm design of the digital SAR processors for SEASAT, SIR-B, ERS-1/2, J-ERS-1, and RADARSAT, as well as several airborne radar systems. He has also worked on systems for processing polarimetric and interferometric radar data, and the compression of radar data. In 1993, he joined the Department of Electrical and Computer Engineering, University of British Columbia, Vancouver, BC, Canada, where he holds the NSERC Industrial Research Chair in Radar Remote Sensing. The Radar Remote Sensing laboratory supports a research staff of eight engineers and students, working in the fields of SAR processing, SAR data encoding, satellite SAR two-pass interferometry, airborne along-track interferometry, airborne polarimetric radar classification, and SAR Doppler estimation.

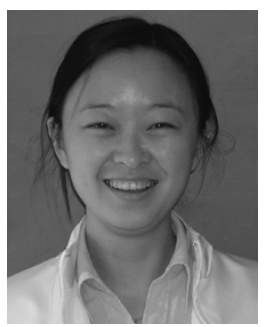

Shu Li received the B.E. degree from the Civil Aviation University of China, Tianjin, in 2000, and the M.S. degree in electrical engineering from the Beijing Institute of Technology, Beijing, China, in 2000 and 2003, respectively. She is currently pursuing the M.A.Sc. degree in the field of satellite SAR Doppler centroid estimation at the University of British Columbia, Vancouver, BC, Canada, in the field of satellite SAR Doppler centroid estimation. 